\title{
Genetic Regulation of Polerovirus and Luteovirus Transmission in the Aphid Schizaphis graminum
}

\author{
M. E. Burrows, M. C. Caillaud, D. M. Smith, E. C. Benson, F. E. Gildow, and S. M. Gray
}

First, third, fourth, and sixth authors: U.S. Department of Agriculture-Agricultural Research Service and Department of Plant Pathology, Cornell University, Ithaca, NY 14853; second author: Biology Department, Ithaca College, Ithaca, NY 14850; and fifth author: Department of Plant Pathology, Pennsylvania State University, University Park 16802.

Accepted for publication 13 March 2006.

\section{ABSTRACT}

Burrows, M. E., Caillaud, M. C., Smith, D. M., Benson, E. C., Gildow, F. E., and Gray, S. M. 2006. Genetic regulation of polerovirus and luteovirus transmission in the aphid Schizaphis graminum. Phytopathology 96:828837.

Sexual forms of two genotypes of the aphid Schizaphis graminum, one a vector, the other a nonvector of two viruses that cause barley yellow dwarf disease (Barley yellow dwarf virus [BYDV]-SGV, luteovirus and Cereal yellow dwarf virus-RPV, polerovirus), were mated to generate F1 and F2 populations. Segregation of the transmission phenotype for both viruses in the $\mathrm{F} 1$ and $\mathrm{F} 2$ populations indicated that the transmission phenotype is under genetic control and that the parents are heterozygous for genes involved in transmission. The ability to transmit both viruses was correlated within the F1 and F2 populations, suggesting that a major gene or linked genes regulate the transmission. However, individual hybrid genotypes differed significantly in their ability to transmit each virus, indicating that in addition to a major gene, minor genes can affect the transmission of each virus independently. Gut and salivary gland associated transmission barriers were identified in the nonvector parent and some progeny, while other progeny possessed only a gut barrier or a salivary gland barrier. Hemolymph factors do not appear to be involved in determining the transmission phenotype. These results provide direct evidence that aphid transmission of luteoviruses is genetically regulated in the insect and that the tissue-specific barriers to virus transmission are not genetically linked.

Additional keywords: circulative nonpropagative transmission, insect genetics, real-time reverse transcription-polymerase chain reaction.
Insects are vectors for a large number of plant and animal viruses (16). There is considerable information available on the genetics determining the mechanism of virus infection and replication cycles, as well as information about how viruses move through the insect host (e.g., barriers, tissue specificity, sites of replication). However, relatively little is known about the genetic control of the transmission process in the insect (18). How insectvirus interactions involving virus recognition by attachment to and endocytotic movement through insect tissues are regulated by insect genes is unknown. Currently, the most common methods to disrupt the virus transmission between hosts are to eliminate the insect or to develop virus resistant hosts. While immune or resistant hosts control the spread of some viruses, this is not an option for most viruses. Attempts to eliminate the vectors remain a widely employed, but often ineffective strategy. Furthermore, increased insecticide use is often associated with increased environmental risk and increased production costs. Therefore, current control options for most viruses transmitted by aphids in a circulative manner are limited. The development of new control strategies utilizing innovative options would be facilitated by an improved understanding of the genetics of virus transmission.

The family Luteoviridae includes the following economically important viruses: Barley yellow dwarf virus (BYDV, luteovirus), Cereal yellow dwarf virus (CYDV, polerovirus), Potato leafroll virus (PLRV, polerovirus), Soybean dwarf virus (luteovirus), and Pea enation mosaic virus (enamovirus). These viruses are trans-

Corresponding author: S. M. Gray; E-mail address: SMG3@ cornell.edu

DOI: 10.1094/PHYTO-96-0828

This article is in the public domain and not copyrightable. It may be freely reprinted with customary crediting of the source. The American Phytopathological Society, 2006. mitted by aphids in a circulative, nonpropagative manner, and many of the virus species are transmitted specifically by one or a limited number of aphid species. The circulative route of virus movement through the aphid body has been well-characterized (11-13). Ingested virions are actively transported across the gut, either hindgut or midgut depending on the virus-aphid combination. Ultrastructural studies indicate that uptake and transport of the virus through the gut is an active receptor mediated endocytosis process, but the putative receptors mediating this process remain to be characterized $(18,29)$. Although the gut has been shown to be a barrier to transmission of CYDV in Metopolophium dirhodum (Walker) (12) and PLRV in Myzus persicae (Sulzer) (45), it does not appear to be the principal barrier in most virusaphid combinations. Aphids that are not vectors can often acquire particles through the gut barrier and retain virus in the hemolymph $(13,44)$.

Once virus particles pass through the hindgut barrier into the hemocoel, little is known about the role of the hemolymph in facilitating or preventing virus transmission. The aphid endosymbiont, Buchnera spp., is known to produce copious amounts of the protein symbionin, a homolog of Escherichia coli GroEL, which binds to luteoviruses and other viruses that are transmitted in a circulative, nonpropagative manner $(1,9,52)$. It has been postulated that when symbionin binds to virus in the hemolymph it may protect the virus from the aphid immune system, which is presumably involved in the recognition of nonself particles in the hemolymph. Little is known about aphid immune responses or about insect immune responses to viruses $(3,18,30,56)$.

Virus particles must move from the hemolymph through the accessory salivary gland in order to be transmitted during feeding $(10,14,37)$. Virus particles pass through the extracellular basal lamina and bind to the basal plasmalemma. Transmissible virions are endocytosed in coated vesicles, fuse with the apical plasma- 
lemma, and are released into the salivary canal (18). The putative receptors regulating virus recognition and transport at the accessory salivary gland have not been characterized.

The aphid Schizaphis graminum (Rondani), commonly known as the greenbug, is a major pest of grain crops and a vector of BYDV and CYDV. It has been a useful model insect for studies on plant resistance, insecticide resistance, and plant responses $(28,41,43)$. A primary attribute is the ability to induce sexual forms in the laboratory facilitating crosses between compatible but genetically different individuals (42). Progeny can be maintained indefinitely as parthenogenetically reproducing genotypes, allowing multiple experiments on genetically identical individuals, an opportunity that does not exist with other insect vectors. Although parthenogenetic reproduction does not ensure complete genetic stability (31), several phenotypic traits, including virus transmission, have been shown to be stable over many generations (40).

The goal of our study was to develop and characterize the F1 and F2 progeny of a cross between two genotypes of $S$. graminum that differ in their ability to transmit the RPV strain of CYDV and the SGV strain of BYDV (34). The hybrid aphid genotypes were used to study the genetic control of virus transmission phenotypes at each step along the circulative pathway and to develop an experimental system that would facilitate the identification of aphid genes and gene products that regulate the circulative transmission of plant viruses. We also used real-time reverse transcriptionpolymerase chain reaction (RT-PCR) to characterize vector and nonvector aphid genotypes for their ability to retain CYDV over time.

\section{MATERIALS AND METHODS}

Aphid genotype maintenance and generation of F1 and F2 crosses. Virus-free genotypes of $S$. graminum, $M$. dirhodum, and Rhopalosiphum padi (L.) were maintained as described by Katsar and Gray (27). The two $S$. graminum genotypes used as parents were described previously (20). Genotype F (Sg-F) (39) was provided by J. Burd (USDA-ARS, Stillwater, OK) and is an efficient vector of BYDV and CYDV (17). Genotype SC (Sg-SC) was collected in South Carolina and is an inefficient vector of BYDV and CYDV (17). To induce sexual forms for mating, aphids colonizing caged barley plants were transferred from growth chambers maintained at $20^{\circ} \mathrm{C}$ (18-h light) to chambers maintained at $15^{\circ} \mathrm{C}$ (12-h light) or $13^{\circ} \mathrm{C}$ (11-h light) and observed weekly for males and sexual females. To make the $\mathrm{F} 1$ cross, virgin female $\mathrm{Sg}-\mathrm{SC}$ were placed in cages with male $\mathrm{Sg}-\mathrm{F}$. Sg-SC does not produce males, and thus a reciprocal cross was not possible. To generate the F2 cross, F1 genotypes were placed in environmental conditions to induce sexual aphids. Resulting virgin females and males were mated randomly. No matings of the same genotype were permitted. Eggs were collected as described by Via (54) and stored for 3 months at 0 to $4^{\circ} \mathrm{C}$. Eggs were placed in dishes containing moist filter paper and fresh barley tissue and incubated at $20^{\circ} \mathrm{C}$. First instar nymphs that hatched and survived were individually transferred to barley plants and allowed to develop parthenogenetically reproducing genotypes that were maintained as described previously (27).

Virus transmission assays. Oat (Avena byzantina K. Koch cv. Coastblack) plants inoculated with RPV or SGV 4 to 5 weeks prior were used as source tissue. Transmission assays were performed as described by Gray et al. (20). Transmission tests for each F1 genotype were repeated three to five times over a 12-month period. Transmission tests for each F2 genotype were repeated two to four times over a 6-month period. Transmission efficiency was calculated as the percentage of plants infested with viruliferous aphids that become infected.

Microinjection of virus into aphids. To determine if the barrier to transmission was associated with virus movement across the hindgut, purified RPV was injected directly into the hemocoel of the parental genotypes, $\mathrm{Sg}-\mathrm{F}$ and $\mathrm{Sg}-\mathrm{SC}$, as well as three $\mathrm{F} 1$ and nine F2 genotypes that were inefficient vectors. If the hindgut was a transmission barrier, we expected the virus transmission efficiency to be increased following virus injection relative to the transmission efficiency following an acquisition feeding period on infected plants. Purified RPV (25) was diluted to a concentration of $40 \mu \mathrm{g} / \mathrm{ml}$ in $0.1 \mathrm{M}\left(\mathrm{K}_{2} \mathrm{H} / \mathrm{NaH}_{2}\right) \mathrm{PO}_{4}$ and injected directly into the hemocoel of the aphid. SGV was not used for injection studies due to the difficulty in obtaining sufficient quantities of purified virus. Fourth instar or apterous adult aphids were immobilized on a small vacuum tube, and less than $0.1 \mu \mathrm{l}$ of purified virus was injected into the hemocoel dorsally at the thorax-abdomen junction using needles drawn from glass microcapillary tubes and attached to a Pico-injector apparatus (PLI-100, Medical Systems Corp., Greenvale, NY). Injected aphids were allowed to recover in plastic dishes containing moist filter paper for ca. $1 \mathrm{~h}$ before being transferred to healthy oat seedlings for a 4- to 5-day inoculation access period (IAP). Five aphids were transferred to each of 8 to 12 oat plants in each treatment. Following the IAP, plants were fumigated, moved to a greenhouse, and observed for the development of virus symptoms for 4 to 6 weeks. Asymptomatic plants and plants expressing mild symptoms were tested by double-antibody sandwich enzyme linked immunosorbent assay (ELISA). Sg-F was included in all injection experiments as a positive control. Injection experiments were repeated seven to nine times for each F1 genotype and five to seven times for each F2 genotype.

Serial transmission experiments. To investigate the quantity of virus acquired and retained by aphids that differ in their ability to transmit RPV, a series of serial transmission experiments were undertaken. Two nonvectors, Sg-SC and M. dirhodum, and two vectors, $\mathrm{Sg}-\mathrm{F}$ and $R$. padi, were compared. Source tissue was cut into 3- to 4-cm sections and randomly sorted into covered plastic dishes. Fourth instar and adult apterous aphids were placed in the dishes and allowed a 48-h acquisition access period. Five aphids of each species were randomly chosen and frozen individually at $-80^{\circ} \mathrm{C}$ for real-time RT-PCR (described below). The remaining aphids were caged, five per plant, on 6-day-old oat seedlings, allowed a 48-h IAP, and the process was repeated until all of the aphids were sampled or deceased. Oat plants were fumigated, placed in the greenhouse, and observed for symptom development as described previously.

Quantification of virus in whole aphids and in aphid hemolymph. In an attempt to quantify the efficiency of virus movement through the hindgut into the hemolymph of vector and nonvector $S$. graminum genotypes, real-time RT-PCR (described below) was used to determine the relative amounts of virus ingested by individual aphids and the amount of virus accumulating in the aphid hemolymph. $R$. padi and $M$. dirhodum were used as controls for these experiments. $R$. padi is an efficient vector of RPV and the hindgut is not a barrier to transmission (12). M. dirhodum is not a vector of RPV and the hindgut was reported to be the principal barrier to transmission (12).

Aphids were fed on RPV-infected or healthy oats for $48 \mathrm{~h}$. Hemolymph samples were obtained by removing all six legs from an aphid. Legs contain hemolymph, are easily collected, and represent a standardized sampling unit that is unlikely to be contaminated by virus from the gut. The remainder of the aphid body was sampled to confirm the aphids fed on source tissue and to determine the total amount of virus ingested. Any drops of hemolymph that exuded from the body as the legs were removed were included with the body, not the legs, for consistency of sampling. The legs and the body were placed in separate tubes containing $50 \mu \mathrm{l}$ of diethylpyrocarbonate (DEPC)-treated water and flash frozen in liquid nitrogen and stored at $-80^{\circ} \mathrm{C}$. Tools used to collect the legs, including a paintbrush and tweezer, were cleaned between each sample by vigorously agitating them in a solution 
of $5 \%$ lauryl sulfate and then in a solution of $10 \%$ bleach $(5.25 \%$ sodium hypochlorite stock solution). Tools were rinsed in distilled water and dried with a clean kimwipe. Parafilm was used as a clean surface on the microscope stage and changed between each aphid genotype. The legs and bodies of 10 individual aphids that had fed on RPV-infected tissue were collected for each aphid genotype in each experiment. Two aphids that had fed on healthy tissue were included in each experimental treatment as negative controls.

RNA extraction and cDNA transcription. A modified trizol extraction procedure was used to extract total RNA from whole aphids or aphid legs. Two hundred microliters of Tri-Reagent (Molecular Research Center, Inc., Cincinnati, $\mathrm{OH}$ ) was added to the frozen legs or body and the mixture was allowed to thaw on ice and then homogenized with an RNase-free pellet pestle (Kontes, Vineland, NJ). The solution was vortexed and incubated at room temperature for $5 \mathrm{~min}$. Forty microliters of chloroform was added and the solution was vortexed and incubated at room temperature for 2 to $3 \mathrm{~min}$. Following centrifugation at $10,000 \times \mathrm{g}$ at $4^{\circ} \mathrm{C}$ for $15 \mathrm{~min}$, the supernatant was transferred to a new tube. One hundred microliters of isopropanol and $25 \mu \mathrm{g}$ of glycogen (Ambion, Austin, TX) were added to precipitate the nucleic acid, and the solution was briefly vortexed. Samples were stored overnight at $-20^{\circ} \mathrm{C}$ and then centrifuged at $16,000 \times g$ for $30 \mathrm{~min}$ at $4^{\circ} \mathrm{C}$. Pellets were washed with $70 \%$ ethanol, dried, and resuspended in $50 \mu \mathrm{l}$ of DEPC-treated water (aphid bodies) or $8 \mu \mathrm{l}$ of DEPC-treated water (aphid legs). Prior to reverse transcription, the nucleic acid samples were treated with DNase to remove residual genomic DNA (DNA-free, Ambion) according to manufacturer instructions. To generate cDNA, 3 or $8 \mu$ of RNA extracted from the aphid bodies or aphid legs, respectively, was added to a $20-\mu \mathrm{l}$ reverse transcription reaction with oligo (dT) and random hexamer primers as provided in the iScript cDNA Synthesis Kit (Bio-Rad Laboratories, Hercules, CA). The cDNA reactions were incubated for $5 \mathrm{~min}$ at $25^{\circ} \mathrm{C}, 30 \mathrm{~min}$ at $42^{\circ} \mathrm{C}$, and $5 \mathrm{~min}$ at $85^{\circ} \mathrm{C}$. The real-time PCR step used $3 \mu \mathrm{l}$ of cDNA from the whole aphid reactions or $8 \mu \mathrm{l}$ of cDNA from the aphid leg reactions in a $25-\mu \mathrm{l}$ reaction creating a cDNA population representing all RNAs including viral RNA.

Real-time RT-PCR. To quantify the amount of RPV ingested by an aphid and/or moving across the hindgut into the hemocoel, the amount of viral cDNA represented in the total cDNA pool was specifically amplified using real-time PCR. Primer and TaqMan probe sequences were designed using RPV GenBank sequences L25299 and NC_004751 with the assistance of Beacon Designer 3.0 (PREMIER Biosoft International, Palo Alto, CA) and validated (5) using Lasergene software (DNASTAR, Inc., Madison, WI). The best set of primers and probe (5) was chosen from the coat protein region (3744 to $4364 \mathrm{bp}$ ). These do not recognize sequences from other species of BYDV, including PAV (accessions X07653, X56050, and NC_002160) and SGV (accessions U06865 and U06866). The forward primer (RPVFp3) sequence was $5^{\prime 4176}$-AATCAATAAGTTCACAATCACCAA-3'. The reverse primer (RPVRp3) sequence was $5^{\prime 4277}$-CACCGTTCCCTTTGTAAAGAA-3'. The TaqMan probe RPVTmp3 $\left(5^{\prime}\right.$-FAM- $-{ }^{4210}$ CGGAGCTTCCCAGCGAAGATGATC-TAMRA-3') was labeled on the $5^{\prime}$ end with fluorescent reporter dye FAM and quenched with TAMRA (Integrated DNA Technologies, Coralville, IA) on the 3' end. PCR analyses were performed according to manufacturer instructions with the iQ Supermix (Bio-Rad), except that PCR volume was $25 \mu \mathrm{l}$. Real-time PCR parameters including annealing temperature and concentrations of primers $(200 \mathrm{nM}$ forward and reverse) and probe $(100 \mathrm{nM})$ were optimized to give $>90 \%$ reaction efficiency (data not shown). The PCRs were cycled in an iCycler (Bio-Rad) using the following parameters: 1 cycle at $95^{\circ} \mathrm{C}$ for $3 \mathrm{~min}, 40$ cycles at $95^{\circ} \mathrm{C}$ for $15 \mathrm{~s}$, and $62^{\circ} \mathrm{C}$ for $1 \mathrm{~min}$. Since there was little variation between wells when run in triplicate in the initial experiments, duplicate wells were run for the majority of the assays to reduce overall costs. A water control was included on each plate. Healthy aphid controls were completed for each genotype. Aphids spiked with known quantities of RPV exhibited 0.4 to $1.4 \%$ variation in the amount of RPV detected using real-time RT-PCR. The total amount of RNA extracted from individual aphids or aphid legs was too small to be quantified with a spectrophotometer, so the amount of variation in extraction efficiency between samples was not determined.

Real-time PCR products were initially run on gels to check for the specificity of the expected 122-bp product. Products obtained from viruliferous aphids were also sequenced to determine that only the RPV sequence was being amplified. No contaminating aphid or plant sequences were observed on the gels or in the sequenced products (data not shown).

Quantification of RPV in aphids and hemolymph samples. An absolute quantification method (6) was used to determine the amount of RPV RNA in each sample. Briefly, the 122-bp PCR product was gel-purified (Ultra Clean 15 DNA Purification Kit, MO BIO Laboratories, Inc., Solana Beach, CA) and a T7 promoter was ligated onto the PCR fragment with the Lign'Scribe Kit (Ambion). An additional PCR was performed to amplify the ligated PCR product, and then RNA was transcribed with the MAXIscript Kit (Ambion). RNA transcripts were treated with DNAse, precipitated twice, and quantified with a spectrophotometer. Transcripts were aliquoted in 5 - $\mu$ l volumes and stored at $-80^{\circ} \mathrm{C}$. A standard curve of at least six dilutions, which encompassed the linear range of the samples, was generated from viral RNA transcripts and included on each PCR plate, along with a water control (6). The concentration of RPV within each sample was calculated by the iCycler iQ Optical System Software version 3.1 (Bio-Rad) and adjusted for dilution. The assay could reliably detect a minimum of 500 copies of RPV in a PCR sample (data not shown).

To determine the reproducibility of the real-time assay, two separate cDNAs were generated from the same RNA extraction for each aphid in the serial transmission experiments. These were consistently parallel and the means overlapped. Therefore, these data were combined and treated as two separate replications of the experiment for statistical analysis. RNA did not degrade significantly over time, as generation of cDNA from the same RNA samples after 3 months gave the same results as generation of cDNA shortly after extraction. Only one cDNA was prepared for the hemolymph experiments due to limited amount of source tissue and the fidelity shown in previous experiments.

Statistical analysis. Analysis of virus transmission data was performed with the Statistical Analysis System version 9.1 (SAS Institute Inc., Cary, NC). Means and standard errors were generated using PROC MEANS. Raw transmission efficiency data were transformed with $\operatorname{arcsine}(x)$. Genetic variability among F1 and F2 genotypes was examined by analysis of variance using PROC MIXED with aphid genotype as a random factor. The genetic correlation between RPV and SGV transmission was estimated separately in the F1 generation and the F2 generation, as product-moment correlations of genotype means, $r_{c m}$ (55) using PROC CORR. The transmission efficiency of RPV and SGV for individual genotypes was compared using a scaled chi-square test (32). For the serial transmission experiments, the concentration of RPV in individual aphid bodies was $\log _{10}$-transformed and the slopes generated with PROC REG. Slopes were compared using PROC GLM.

\section{RESULTS}

F1 genotypes and virus transmission efficiency. Two hundred and fifty-one fertilized eggs were collected from a cross between female $\mathrm{Sg}-\mathrm{SC}$ and male $\mathrm{Sg}-\mathrm{F}$. Of these eggs, 46 hatched and a total of 13 F1 hybrid nymphs survived to establish parthenogenetically reproducing genotypes. 
The mean transmission efficiency of RPV and SGV for the F1 hybrid population was 45 and $41 \%$, respectively, and intermediate between the two parents, Sg-SC (0\%) and Sg-F (84\% RPV and $79 \% \mathrm{SGV}$ ) (Fig. 1A). There was significant variation among the F1 genotypes for the transmission of RPV ( 0 to $83 \%, F_{12.51}=$ 9.66, $P<0.001)$ and SGV ( 2 to $\left.90 \%, F_{12,40}=6.29, P<0.001\right)$, indicating the parents are not fixed for all loci involved in transmission. The RPV transmission efficiency for five of the F1 genotypes (F1-2, F1-4, F1-5, F1-9, and F1-10) was similar to the transmission competent parent $(\mathrm{Sg}-\mathrm{F})$, whereas three genotypes (F1-1, F1-8, and F1-13) were similar to the nonvector parent, SgSC. The remaining F1 genotypes exhibited RPV transmission efficiencies intermediate between the parents (Fig. 2). The transmission efficiency of RPV and SGV was correlated in the F1 $\left(\mathrm{r}_{\mathrm{cm}}=0.59, P<0.001\right)$ (Fig. 3A), suggesting that one major gene or a set of closely linked genes affects transmission of both viruses. However, the F1 genotypes did not always behave similarly for the transmission efficiency of both viruses. Six of the F1 genotypes (F1-3, F1-7, F1-9, F1-10, F1-11, and F1-12) differed significantly $(P \leq 0.05)$ in their ability to transmit RPV and SGV (Fig. 2).

F2 genotypes and virus transmission efficiency. Eleven of the thirteen F1 genotypes were reared under the environmental conditions that induced sexual forms in the parents. Five of the F1 genotypes (F1-1, F1-4, F1-5, F1-9, and F1-11) produced males,

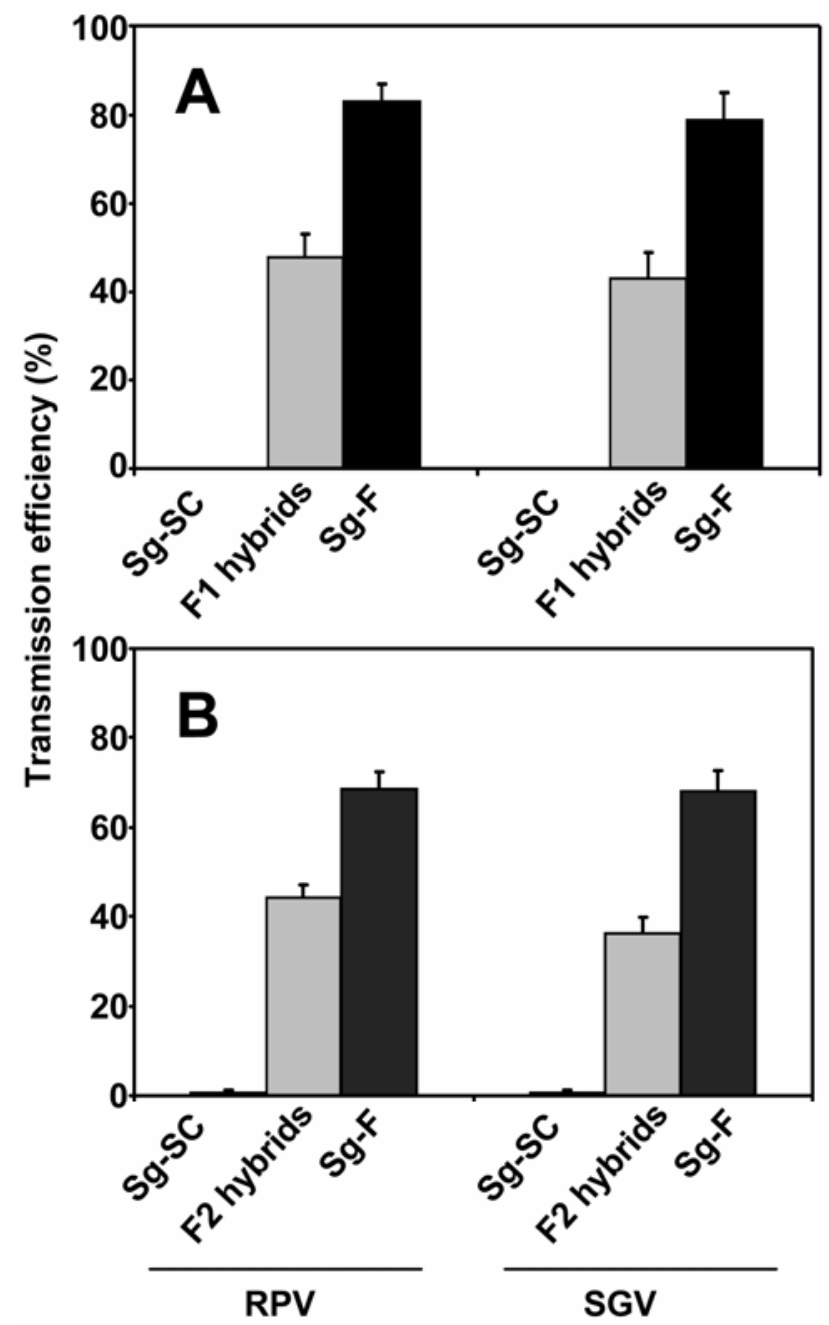

Fig. 1. The mean transmission efficiency of Cereal yellow dwarf virus-RPV and Barley yellow dwarf virus-SGV by the Schizaphis graminum parental genotypes, nonvector genotype SC (Sg-SC), and vector genotype $\mathrm{F}(\mathrm{Sg}-\mathrm{F})$ and their progeny A, $13 \mathrm{~F} 1$ hybrid genotypes and B, 51 F2 hybrid genotypes. Error bars indicate the standard error of the mean. and nine of the F1 genotypes (F1-1, F1-2, F1-3, F1-4, F1-5, F1-7, F1-9, F1-10, and F1-11) produced females. Genotypes F1-6 and F1-8 did not produce any sexuals. Males and females were randomly mated to produce an F2 generation. One thousand ninetythree fertilized eggs were collected from multiple random crosses, 209 eggs hatched, and 51 individual F2 genotypes produced parthenogenetic reproducing genotypes.

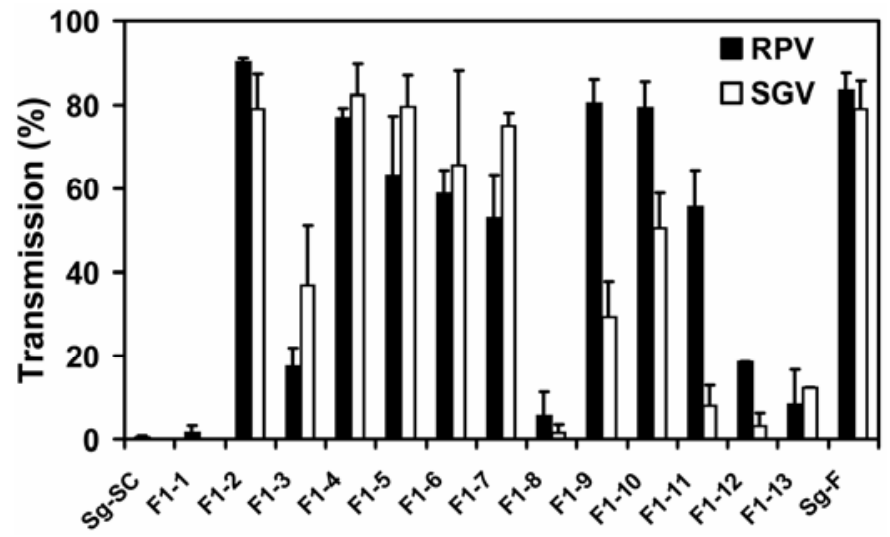

Fig. 2. The transmission efficiency of Cereal yellow dwarf virus-RPV and Barley yellow dwarf virus-SGV by Schizaphis graminum genotype SC ( $\mathrm{Sg}$ $\mathrm{SC}) \times S$. graminum genotype $\mathrm{F}(\mathrm{Sg}-\mathrm{F}) \mathrm{F} 1$ genotypes. The parents and designation for each Sg-SC $\times \mathrm{Sg}-\mathrm{F} \mathrm{F1}$ genotype is noted on the $x$ axis. After a 48-h acquisition access period on source tissue, five fourth instar or adult aphids were placed on each of 16 oat plants and allowed a 5-day inoculation access period. Bars represent the mean of two to five replicates. Error bars indicate the standard error of the mean.
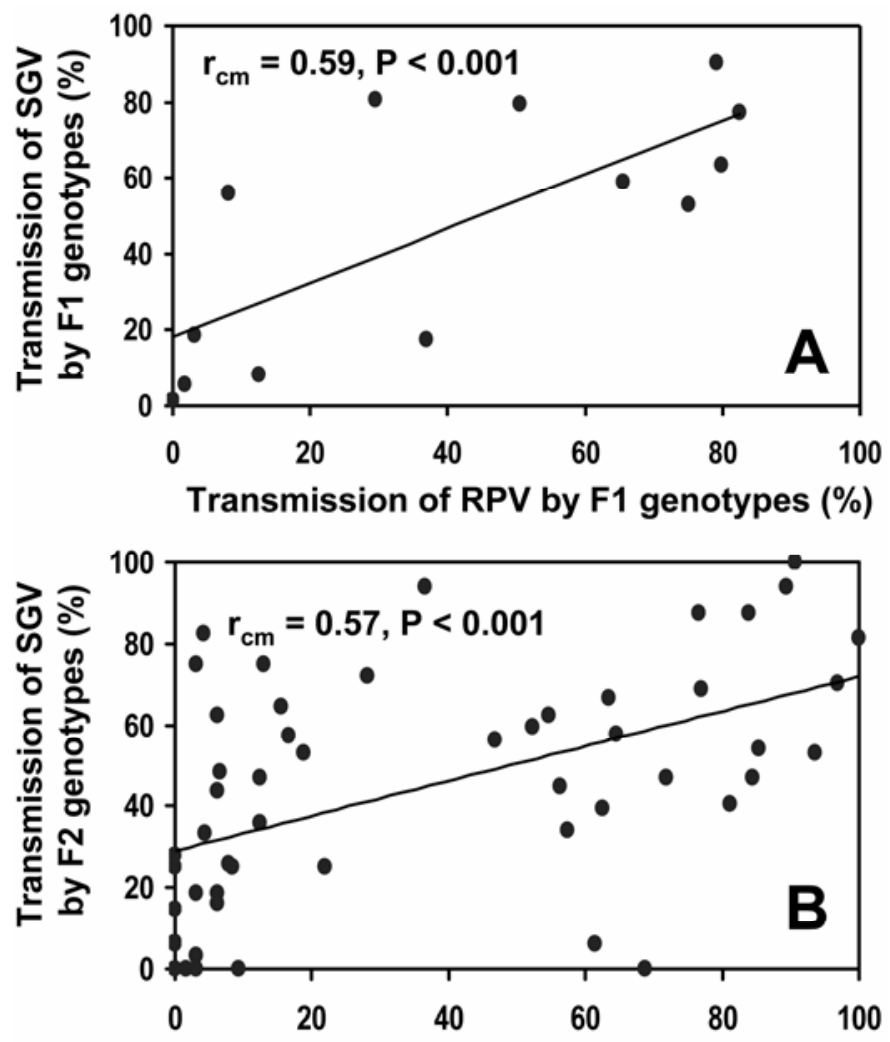

Transmission of RPV by F2 genotypes (\%)

Fig. 3. The genetic correlation of Cereal yellow dwarf virus-RPV and Barley yellow dwarf virus-SGV transmission by $\mathbf{A}, \mathrm{F} 1$ and $\mathbf{B}, \mathrm{F} 2$ genotypes from a cross between nonvector Schizaphis graminum genotype $\mathrm{SC}(\mathrm{Sg}-\mathrm{SC})$ and vector $S$. graminum genotype F (Sg-F). The genetic correlation was estimated as product-moment correlations of genotype means (the $r_{c m}$ of Via [55]). 
Similar to the F1 population, there was significant variation in transmission efficiency of RPV and SGV among the F2 genotypes (RPV: $F_{52,155}=3.26, P<0.001 ; \mathrm{SGV}: F_{52,155}=4.4, P<0.001$ ) (Fig. 4). Transmission efficiency for RPV and SGV ranged from 0 to $100 \%$. The mean transmission efficiency was $45 \%$ for RPV and $41 \%$ for $\mathrm{SGV}$, and there was no directional dominance toward either parental genotype (Fig. 1B). Again, the transmission efficiency of RPV and SGV was correlated $\left(\mathrm{r}_{\mathrm{cm}}=0.57, P<0.001\right)$ (Fig. 3B); however, the majority of F2 genotypes differed significantly in their ability to transmit RPV and SGV $(P<0.05,30$ of 51 genotypes).

Barriers to transmission in $S$. graminum genotypes. Injection of purified RPV into Sg-F resulted in transmission efficiencies similar to those when the aphids acquired virus by feeding on infected tissue (Tables 1 and 2). While acquisition of virus from feeding on infected tissue has rarely resulted in $\mathrm{Sg}-\mathrm{SC}$ transmitting virus in hundreds of trials over the past 5 years, injection of purified RPV into the hemocoel increased the mean transmission efficiency from 0 to $15 \%$ (Table 1 ). These results indicate that if higher concentrations of the virus accumulate in the hemocoel, i.e., move across the hindgut barrier, then virus movement through the accessory salivary glands can occur, albeit at a lower rate of efficiency than in the vector, $\mathrm{Sg}-\mathrm{F}$.

Similar to the results with $\mathrm{Sg}-\mathrm{SC}$, injection of purified virus into the hemocoel of the three F1 genotypes (F1-1, F1-3, and F18), which were inefficient vectors of RPV (Fig. 2), resulted in only slight increases in transmission efficiency relative to feeding assays (Table 1). The accessory salivary gland is the principal barrier to transmission of RPV in these genotypes.

Injection of purified virus into three F2 genotypes (F2-E1, F2G5, and F2-I2) with intermediate levels of RPV transmission efficiency (25 to $40 \%)$ relative to $\mathrm{Sg}-\mathrm{F}(67 \%)$ and $\mathrm{Sg}-\mathrm{SC}(<1 \%)$

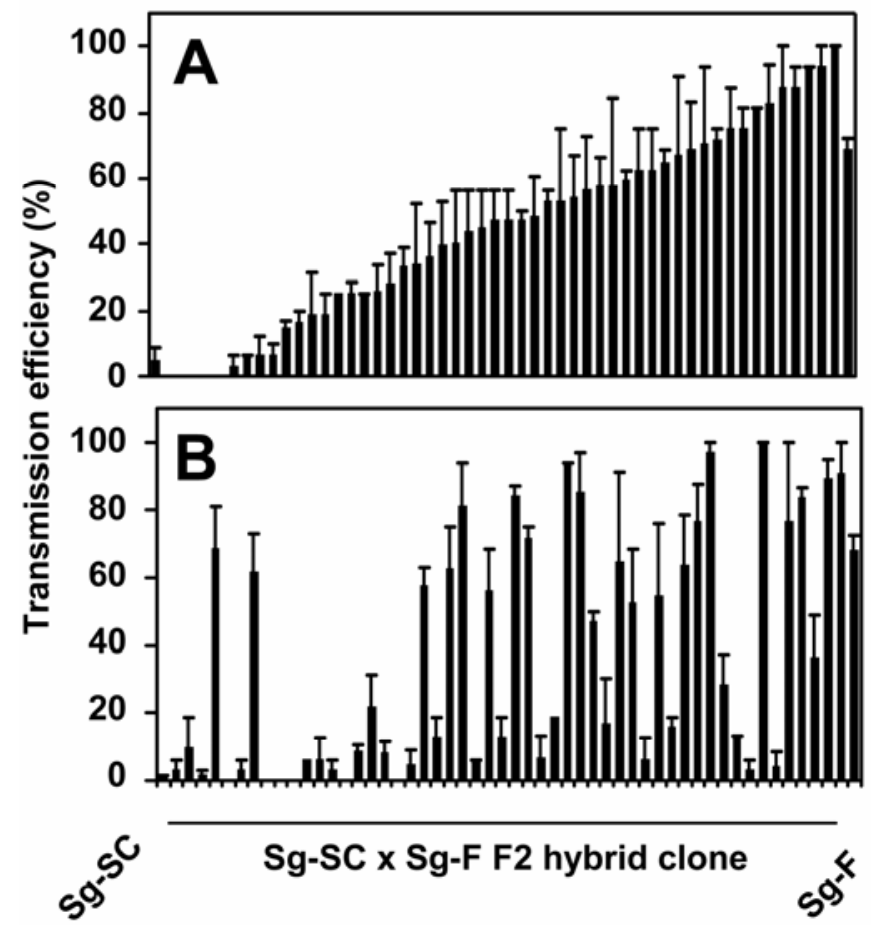

Fig. 4. The transmission efficiency of Schizaphis graminum genotype SC (Sg$\mathrm{SC}) \times S$. graminum genotype $\mathrm{F}(\mathrm{Sg}-\mathrm{F}) \mathrm{F} 2$ hybrid genotypes after feeding acquisition of A, Cereal yellow dwarf virus-RPV (RPV) and B, Barley yellow $d$ warf virus-SGV. The transmission phenotypes of the original parents ( $\mathrm{Sg}-\mathrm{SC}$ and $\mathrm{Sg}-\mathrm{F}$ ) are noted on the $x$ axis, and $\mathrm{Sg}-\mathrm{SC} \times \mathrm{Sg}-\mathrm{F}$ F2 genotypes are arranged from left to right in both $\mathbf{A}$ and $\mathbf{B}$ in order of increasing efficiency of RPV transmission. After a 48-h feeding acquisition on infected source plants, five fourth instar or adult aphids were placed on each of 16 oat plants and allowed a 5-day inoculation access period. Bars represent the mean of two to four replicates. Error bars indicate the standard error of the mean. also did not significantly increase the transmission efficiency (Table 2). However, injection of purified RPV into the hemocoel of six F2 genotypes (F2-C1, F2-C2, F2-D2, F2-K2, F2-M1, and $\mathrm{F} 2-\mathrm{P} 1)$ that were inefficient vectors similar to $\mathrm{Sg}-\mathrm{SC}$ did result in increased transmission efficiency with mean transmission efficiency ranging from 19 to $55 \%$ (Table 2). This suggests that hindgut does play a role in reducing the movement of ingested virus into the hemocoel of these genotypes. The transmission efficiency of genotype F2-C2 following virus injection was comparable to the competent parent $\mathrm{Sg}-\mathrm{F}$, suggesting that the hindgut is the principal barrier to virus movement in this genotype.

Real-time RT-PCR of aphid hemolymph. To further test the hypothesis that the hindgut can restrict the movement of virus into the hemocoel, relative amounts of ingested virus and acquired virus, i.e., virus moving into the hemocoel, were determined using a real-time RT-PCR assay. Virus levels were measured in three inefficient vector genotypes of $S$. graminum: F2-C2, which appeared from the injection experiment results to have an active hindgut barrier, and $\mathrm{Sg}-\mathrm{SC}$ and $\mathrm{F} 2-\mathrm{K} 2$, which appear to have less active hindgut barriers. We also used another inefficient vector of RPV, $M$. dirhodum, that was reported to have a hindgut barrier to virus movement (12). $\mathrm{Sg}-\mathrm{F}$ and $R$. padi served as positive controls; both are efficient vectors of RPV that would not be expected to have any measurable hindgut barrier to virus movement.

Within a given experiment, the amount of virus in the bodies of the various aphid species and genotypes was comparable (Fig. 5). This indicates that all aphids ingested similar amounts of virus and that feeding behavior or ability to extract virus from infected phloem tissue did not contribute to differences in transmission efficiency. Analysis of the data revealed a significant experimenttreatment interaction $(P=0.002)$ indicating that, as expected, the amount of virus acquired by each genotype varied between experiments. This can be attributed to several factors including the quality and virus distribution within the source tissue, and feeding activity of individual aphids during the 48-h acquisition access period. This interaction prevented pooling of the data across experiments.

Virus was detected in the hemolymph of all aphid species and genotypes albeit at several orders of magnitude less than in the bodies. Mean levels of virus in hemolymph was similar for most of the aphid species and genotypes regardless of their vector competency with the exception of genotype F2-C2, which consistently had a significantly lower amount of virus in the hemolymph. Furthermore, in 11 of the 30 individual F2-C2 aphids tested over

TABLE 1. A comparison of transmission efficiency of parents and Sg-SC $\times$ $\mathrm{Sg}-\mathrm{F} \mathrm{F} 1$ hybrids after injection of purified Cereal yellow dwarf virus (CYDV)-RPV into the aphid hemocoel or feeding on source plants infected with CYDV-RPV ${ }^{\mathrm{a}}$

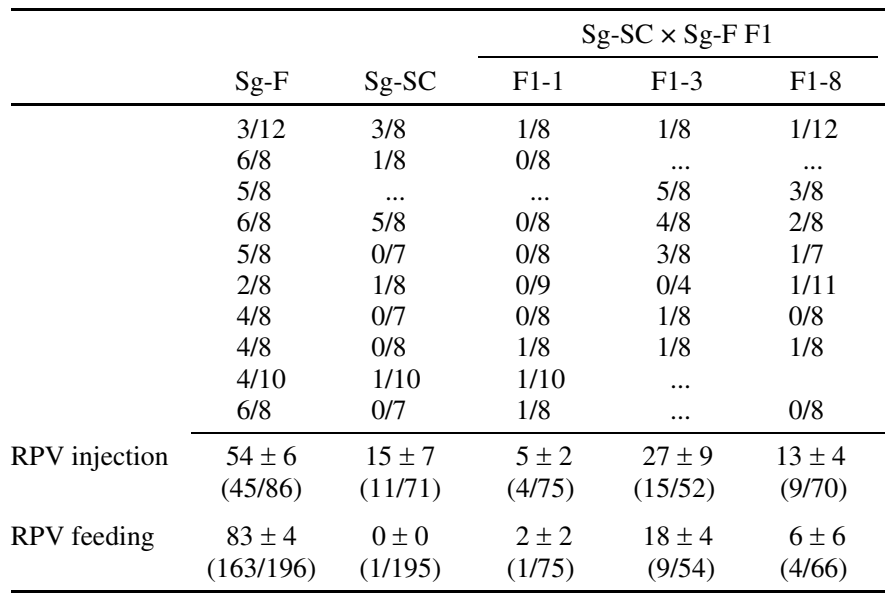

${ }^{\text {a }}$ Mean percent transmission \pm standard error. The number of plants infected with virus/the number of plants infested with viruliferous aphids (five aphids per plant) is in parentheses. 
the three experiments, the level of virus in the hemolymph was below the level of detection of the assay (ca. 500 copies). These aphids were not included in the calculation of the means shown in Figure 5. Virus levels below the detection limit were only found in 9 of the 130 other aphids examined in these experiments.

Acquisition and retention of RPV after feeding acquisition. The experimental evidence suggests that the hindgut is not a principal barrier to virus movement in most of the inefficient vector genotypes. Therefore, in the aphid genotypes with low virus transmission efficiency, either the virus must be blocked from movement into the accessory salivary glands or it is degraded or inactivated in the hemolymph. In an attempt to quantify the rate of virus loss from vectors and nonvectors over time, the amount of virus acquired and retained was measured using real-time RTPCR in two nonvector aphids, M. dirhodum and Sg-SC, and two vector aphids, $R$. padi and Sg-F. All aphid species and genotypes, regardless of vector status, acquired and retained virus over the course of the experiment (Fig. 6). The rate of virus degradation or loss due to feeding as measured by the slope of the regression lines was similar for all four aphids and in both experiments $(P>$ $0.500)$. The vector species continued to transmit virus near to the end of the experiment (Table 3). Although the transmission effi-

TABLE 2. A comparison of transmission efficiency of parents and Sg-SC $\times$ Sg-F F2 hybrids after injection of purified Cereal yellow dwarf virus (CYDV)-RPV into the aphid hemocoel or feeding on source plants infected with CYDV-RPV ${ }^{\text {a }}$



a Mean percent transmission \pm standard error. Calculated as the number of plants infected with virus / the number of plants infested with viruliferous aphids (five aphids per plant) in parentheses.

${ }^{b}$ Transmission efficiency of F2 clones was classified as inefficient or intermediate after feeding on CYDV-RPV-infected source plants.



Fig. 5. Real-time reverse transcription-polymerase chain reaction (RT-PCR) of bodies and hemolymph from 10 individual aphids of six aphid genotypes: Metopolophium dirhodum, Rhopalosiphum padi, Schizaphis graminum genotype SC (Sg-SC), F (Sg-F), and Sg-SC $\times$ Sg-F F2 hybrid genotypes F2-K2 and F2-C2. Aphids were fed on oat tissue infected with Cereal yellow dwarf virus-RPV for $48 \mathrm{~h}$ before dissection and RNA extraction. Real-time RT-PCR methods are described in the text. Error bars represent the standard error of the mean. 
ciency of vector aphids did decrease at the later time points in most experiments, this was not associated with a decrease in RPV concentration measured within the aphids. The nonvector species did not transmit virus at any time point.

\section{DISCUSSION}

We found that the transmission of the related BYDV-SGV and CYDV-RPV is a genetically controlled, heritable trait in S. grami- num. The genetic control of plant virus transmission by insect vectors was recognized in early studies of virus-insect interactions. Storey (47) was the first to determine that populations of the leafhopper Cicadulina mbila Naude differed in their ability to transmit Maize streak virus (family Geminiviridae, genus Mastrevirus), and phenotypes could be maintained by continued breeding of individuals with the same transmission phenotype. A limitation of most virus-vector genetic studies is the inability to maintain genetically identical populations for extended periods of
M. dirhodum

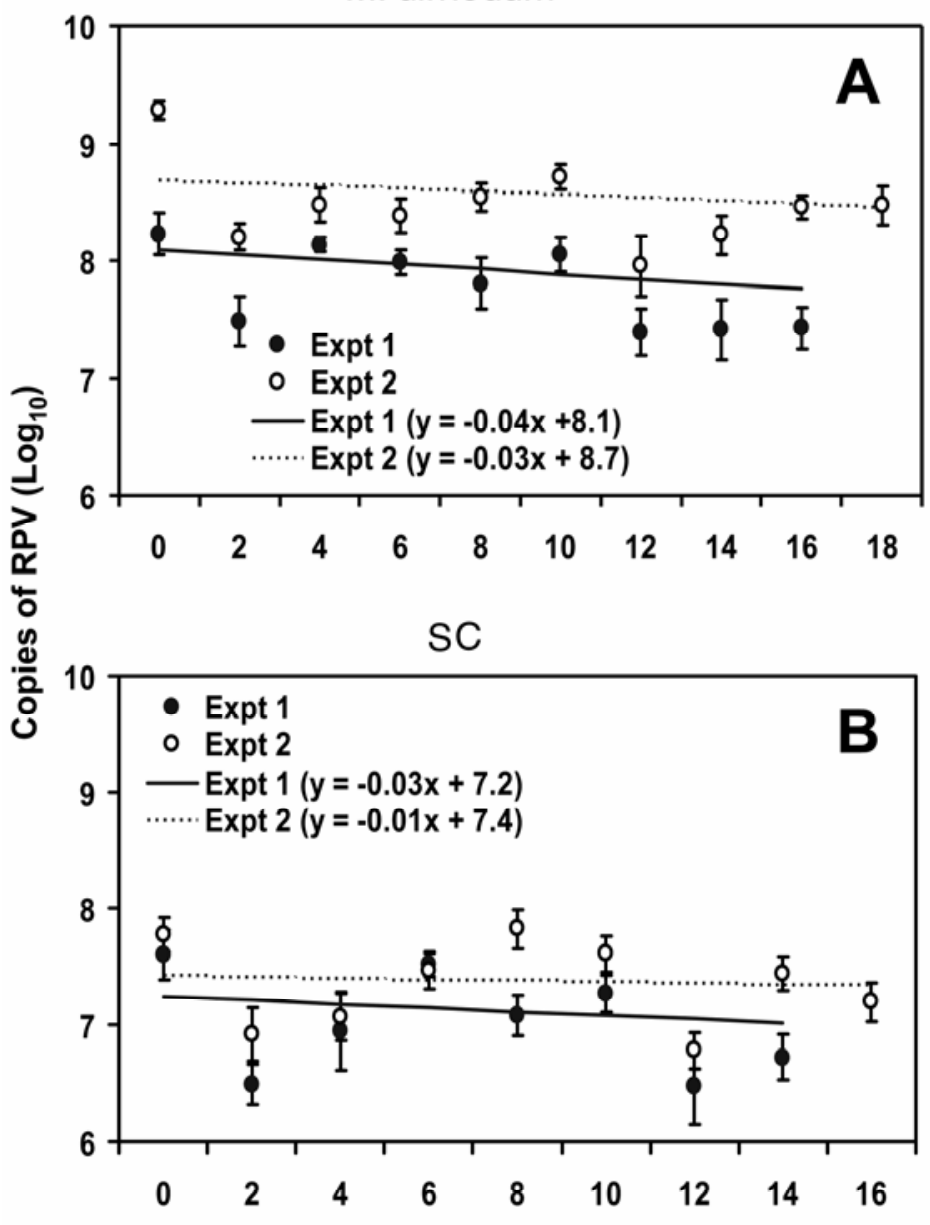

R. padi
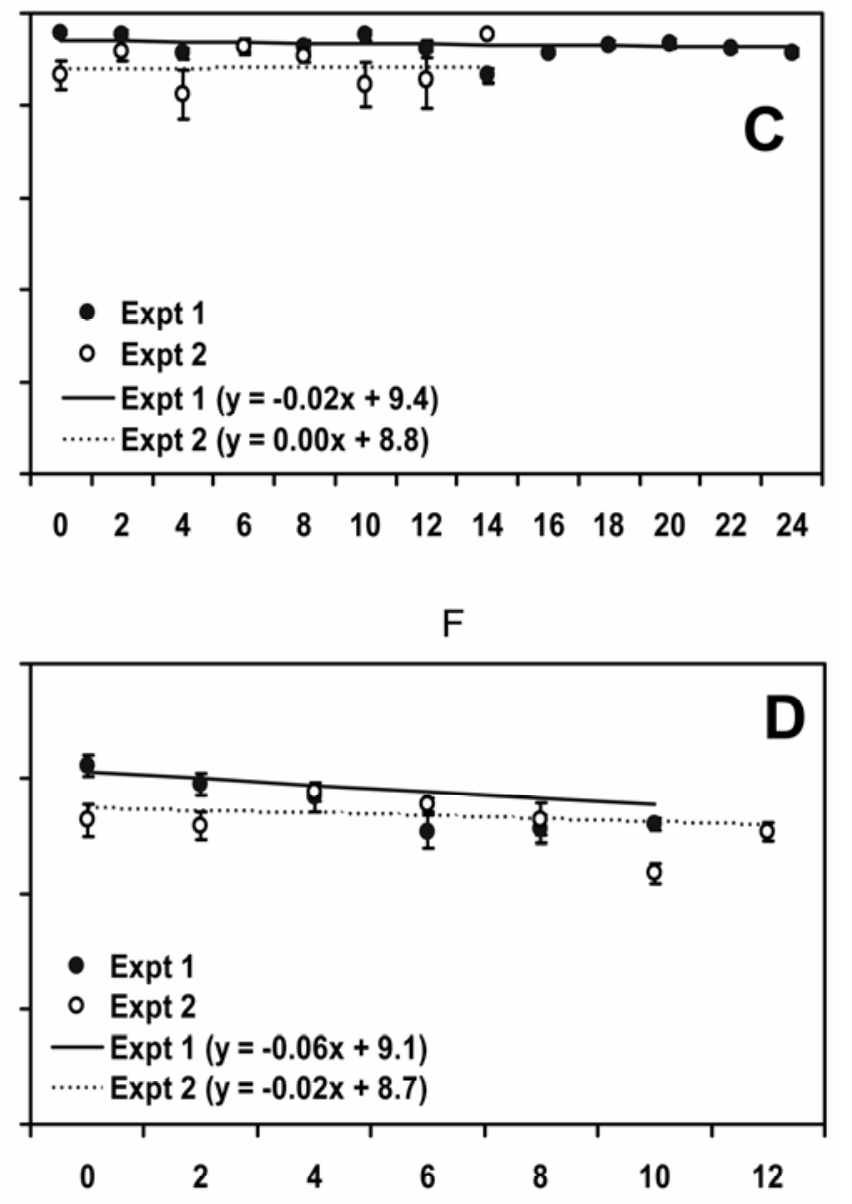

Time (Days)

Fig. 6. Real-time reverse transcription-polymerase chain reaction of aphids fed on Cereal yellow dwarf virus-RPV-infected oat plants for $48 \mathrm{~h}$ and then serially transferred to healthy plants. Nonvector aphids A, Metopolophium dirhodum and B, Schizaphis graminum genotype SC. Vector aphids C, Rhopalosiphum padi and D, Schizaphis graminum genotype F. Five aphids were sampled at each time period and processed individually. An absolute quantification method was used to determine the concentration of RPV. Two independent cDNA reactions were performed and treated as two experimental replicates. Error bars represent the standard error of the mean. Data were $\log _{10}$-transformed before statistical analysis.

TABLE 3. Transmission frequency of Cereal yellow dwarf virus-RPV during serial transmission experiments with Metopolophium dirhodum, Schizaphis graminum clone $\mathrm{SC}$ (Sg-SC), Rhopalosiphum padi, and S. graminum clone F (Sg-F) ${ }^{\mathrm{a}}$

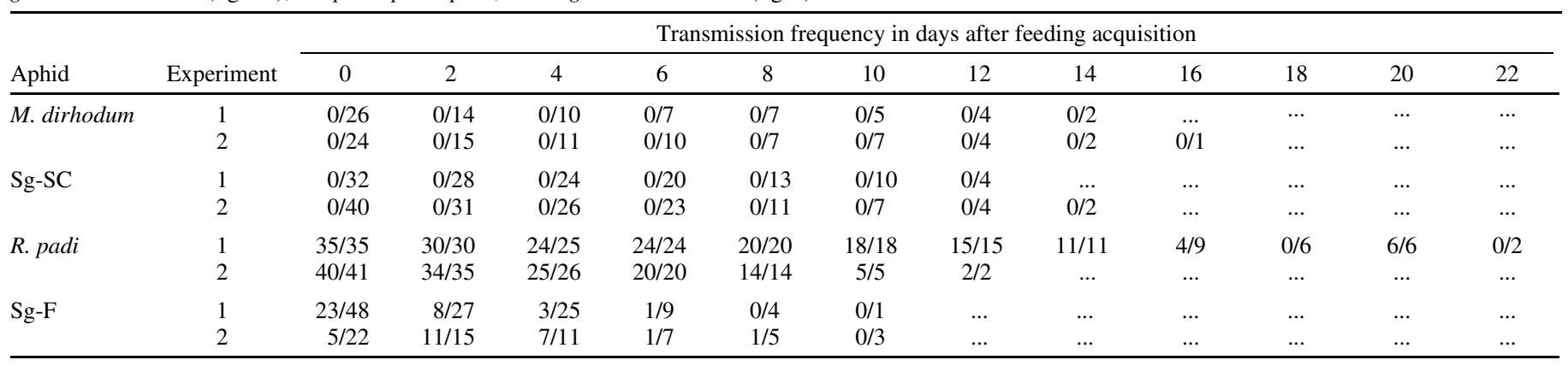

${ }^{a}$ Results are given as the number of positive oat plants per number of plants inoculated with five aphids per plant. 
time. With aphids, we cannot only cross aphid genotypes via sexual recombination, we can maintain genotypes asexually and perform multiple, repeated experiments on the same genotype for an indefinite period. Although there is some evidence of somatic mutations in clonal lineages over time (31), genotypes can be maintained for extended periods with stable transmission phenotypes (40).

Our data indicate that the transmission of luteoviruses by $S$. graminum is a multigenic trait. There was significant genetic variation among the F1 and F2 genotypes for their ability to transmit RPV and SGV. This suggests that the parents are not fixed at all loci involved in transmission, which is expected for field-collected animals (as opposed to animals from inbred colonies in the laboratory). The mean transmission efficiency of the F1 and F2 genotypes in our study was intermediate between that of the mean transmission efficiency of the parents, indicating no directional dominance for either RPV or SGV transmission. This also indicates that transmission phenotype is inherited in an additive, rather than a dominant or epistatic, manner. Papura et al. (36) and Dedryver et al. (7) have investigated the BYDV-PAV transmission phenotype of F1 populations of Sitobion avenae generated from crosses between genotypes that differ in transmission efficiency or from selfed inefficient vectors. Similar to our results, the individual F1 genotypes varied in their ability to transmit BYDV-PAV, allowing them to conclude that transmission phenotype is polygenic, with a degree of additivity among the genes involved (7). In leafhoppers, breeding of individuals with different transmission phenotypes revealed the transmission of maize streak was inherited as a simple dominant Mendelian factor that was linked to the sex of the insect (47). Others have investigated the genetics of circulative nonpropagative and circulative propagative virus transmission in leafhoppers and not all are controlled by a single dominant gene (57). The susceptibility or refractiveness of Aedes spp. and Culicoides spp. to viral diseases has generally been found to involve one major gene, with minor genes affecting the interaction in some cases $(22,26,33,49)$. However, in other systems, vector competence is multigenic $(50,51)$. The inheritance of susceptibility to dengue- 2 virus is different in Aedes albopictus and A. aegypti $(21,22)$. It is clear that the genetics of virus transmission is specific to virus-insect combinations and no generalities can be inferred.

We found a statistical correlation between the transmission of RPV and SGV, suggesting that some of the genes involved in transmission could be shared. However, individual genotypes transmitted RPV and SGV at significantly different rates. We speculate that genes involved in transmission could include protein receptors associated with virus recognition and cellular transport, hemolymph factors, and contributions from endosymbionts, some of which would interact with both RPV and SGV in the same manner, and others that may discriminate between the two. This is the first evidence to our knowledge that the transmission of two related viral species can be controlled by different genes within the same insect genotype, and these genes can be inherited independently. Preliminary estimates of the number of genes involved in our system indicate there are very few aphid genes controlling luteovirus transmission (unpublished data). This suggests that a mapping strategy to identify genes would be successful. The genotypes developed here will be useful in that effort.

The location of the transmission barrier (gut or salivary gland) within the aphid is important for understanding the movement of virus through the aphid and where the genes involved in transmission may function. Storey (48) was the first to demonstrate that transmission-inactive leafhoppers were unable to transmit a circulative virus due to a block in the virus movement from the gut to the hemolymph. In $A$. aegypti, gene candidates for the transmission of Plasmodium and dengue- 2 virus have been identified, many of which are differentially expressed in the gut (15, 35 ). Aphid proteins which bind to luteoviruses in vitro have been isolated and in some cases identified $(9,29,46)$, but it is unknown how they function with respect to virus transmission. We used a combination of injection experiments and real-time RT-PCR to show that transmission barriers present in the parents were separated in the progeny. The vector parent, $\mathrm{Sg}-\mathrm{F}$, is not postulated to contain a barrier to transmission. However, there is evidence to suggest that a partial barrier may exist. First, the transmission of BYDV and CYDV by Sg-F averages 60 to $80 \%$, but does not often approach rates of $100 \%$. RPV transmission rates of $100 \%$ are regularly achieved with the vector $R$. padi (Table 3) (40). Additionally, several F2 genotypes transmitted RPV and SGV at a rate of $100 \%$. Although there were fewer trials for the F2 genotypes than for Sg-F, these results suggest that $\mathrm{Sg}-\mathrm{F}$ is not homozygous for all genes facilitating transmission. A combination of the gut and salivary gland barriers were found to prevent transmission of RPV in the nonvector parent Sg-SC based on the results of injection experiments. The amount of RPV in the hemolymph of Sg-SC could not be distinguished from vector genotypes using real-time RT-PCR, demonstrating the gut is not an absolute barrier.

If the barriers to transmission operating in $\mathrm{Sg}$-SC are under genetic control, then they will likely segregate in the F1 and F2 hybrid populations. In the three F1 genotypes tested and in the majority of the F2 genotypes, injection results were similar to $\mathrm{Sg}$ $\mathrm{SC}$, indicating the principal barrier to transmission was not the gut. A partial, but not complete, barrier to transmission in the gut was identified using injection in genotype F2-C2, and this result was confirmed using real-time RT-PCR. As described previously, we did not have an internal control for hemolymph RNA levels and some of these low levels of virus could have been due to less than optimal RNA extraction or quality, but it is unlikely that this is solely responsible for the low levels of virus detected in the genotype F2-C2. Further experiments with F2-C2 have suggested that the gut barrier is overcome by feeding aphids on highly concentrated, purified RPV (F. Gildow, unpublished data). The gut has been shown to be a barrier to transmission of BYDV-RPV in $M$. dirhodum based on recovery of viable virus from the hemolymph (12) and a barrier to a strain of PLRV in Myzus persicae based on injection of virus into the hemocoel (45). However, the gut does not appear to be a principal barrier in most virus-aphid combinations (12), and we did not observe a gut barrier to the movement of RPV across the gut in $M$. dirhodum in our study using real-time RT-PCR of aphid hemolymph. Real-time RT-PCR is a much more sensitive technique than that used previously (12), and does not distinguish between viable and nonviable virus particles. Additionally, transmission efficiency can be related to the amount of virus acquired from source tissue, and titer within the aphid $(19,38)$. The gut may serve as a rate-limiting barrier to transmission, i.e., allowing virus to pass, but limiting the movement of virus and thus lowering transmission efficiency. This seems to be the case with genotype F2-C2.

The role of the hemolymph in permitting or preventing virus transmission is not known. Previous studies have used immunological techniques (ELISA) to study acquisition and retention of luteoviruses by aphids $(4,8,23,24)$. In these studies, virus is acquired by the insect and immunological detection decreases rapidly over a period of 1 to 2 weeks, suggesting the virus is degraded or otherwise altered. We used real-time RT-PCR to show that aphids acquired and retained virus in their bodies for up to 24 days. A rapid degradation of viral RNA was not detected and since unprotected RNA would not be stable, a degradation of virus particles is not a likely reason to explain the loss of transmission in this system. The transmission of virus by vector aphids decreased slightly over time, but this was not associated with a decrease in virus concentration within the aphid. Note that we were detecting viral RNA, not coat protein. To explain previous immunological results, we can speculate that viral capsids could be altered by factors in the gut or hemolymph, masking the 
epitopes required for antibody recognition. The aphid endosymbiont Buchnera is known to produce symbionin, a homolog of E. coli GroEL, which can bind nonspecifically to luteovirus and other viruses that are transmitted in a similar manner $(1,2,9$, $52,53)$. It has been postulated that symbionin can protect viruses from degradation during movement through the aphid $(52,53)$. The parental genotypes used here and other S. graminum genotypes tested do not harbor known secondary symbionts, although they do contain Buchnera spp. (N. Moran, personal communication). The virus must pass through the gut via formation of vesicles in the apical plasmalemma $(11,12,18)$. This process, or chemical alterations in the hemocoel, could alter the viral capsid structure. Additionally, the aphid immune system is presumably involved in the recognition of nonself particles in the hemolymph and could play a role in sequestering or altering virions. Very little is known about insect immunity, and immunity against viruses in particular $(3,18,30,56)$.

While it has long been suggested that virus transmission was at least partially under genetic control, this study offers direct evidence not only that multiple genetic factors regulate the circulative transmission of plant viruses, but also that different loci regulate the circulative transmission of two related virus species that share a common vector and mechanism of transmission. Furthermore, this is the first example to demonstrate that multiple genetically controlled barriers to circulative transmission can exist within an aphid species and that these different barriers can be isolated using a genetic strategy. Additionally, we have shown that virus is not degraded in the hemolymph as a mechanism of preventing virus transmission by nonvectors. Previous work has demonstrated the utility of $S$. graminum as a workable genetic system for looking at the genetics of host and insecticide resistance $(28,43)$. Our work now demonstrates the utility of this aphid as a model for investigating the genetics and genomics of circulative plant virus transmission. Through these studies, we will be able to better understand the specificity of vector-virus interaction and the evolution of this interrelationship.

\section{ACKNOWLEDGMENTS}

We thank T. Hammond for planting and greenhouse maintenance and J. Booth, Department of Biological Statistics and Computational Biology, Cornell University, for statistical assistance. This project was supported by the National Research Initiative of the USDA Cooperative State Research, Education and Extension Service grant no. 2003-35302-13472 to S. M. Gray and F. E. Gildow.

\section{LITERATURE CITED}

1. Akad, F., Dotan, N., and Czosnek, H. 2004. Trapping of Tomato yellow leaf curl virus (TYLCV) and other plant viruses with a GroEL homologue from the whitefly Bemisia tabaci. Arch. Virol. 149:1481-1497.

2. Baumann, P., Baumann, L., Lai, C. Y., Roubakhsh, D., Moran, N. A., and Clark, M. A. 1995. Genetics, physiology, and evolutionary relationships of the genus Buchnera-intracellular symbionts of aphids. Annu. Rev. Microbiol. 49:55-94.

3. Beerntsen, B. T., James, A. A., and Christensen, B. M. 2000. Genetics of mosquito vector competence. Microbiol. Mol. Biol. Rev. 64:115-137.

4. Bencharki, B., Yamani, M. E., and Zaoui, D. 2000. Assessment of transmission ability of Barley yellow dwarf virus-PAV isolates by different populations of Rhopalosiphum padi and Sitobion avenae. Eur. J. Plant Pathol. 106:455-464.

5. Boeckman, F., Brisson, M., and Tan, L. 2003. Real-time PCR: General considerations. Technical note 2593. Bio-Rad Laboratories, Hercules, CA.

6. Bustin, S. A. 2000. Absolute quantification of mRNA using real-time reverse transcription polymerase chain reaction assays. J. Mol. Endocrinol. 25:169-193.

7. Dedryver, C. A., Riault, G., Tanguy, S., Le Gallic, J. F., Trottet, M., and Jacquot, E. 2005. Intra-specific variation and inheritance of BYDV-PAV transmission in the aphid Sitobion avenae. Eur. J. Plant Pathol. 111:341-354.

8. Fargette, D., Jenniskens, M.-J., and Peters, D. 1982. Acquisition and transmission of pea enation mosaic virus by the individual pea aphid. Phytopathology 72:1386-1390.
9. Filichkin, S. A., Brumfeld, S., Filichkin, T. P., and Young, M. J. 1997. In vitro interactions of the aphid endosymbiotic SymL chaperonin with Barley yellow dwarf virus. J. Virol. 71:569-577.

10. Gildow, F. E. 1982. Coated-vesicle transport of luteoviruses through salivary glands of Myzus persicae. Phytopathology 72:1289-1296.

11. Gildow, F. E. 1985. Transcellular transport of barley yellow dwarf virus into the hemocoel of the aphid vector, Rhopalosiphum padi. Phytopathology 75:292-297.

12. Gildow, F. E. 1993. Evidence for receptor-mediated endocytosis regulating luteovirus acquisition by aphids. Phytopathology 83:270-277.

13. Gildow, F. E., and Gray, S. M. 1993. The aphid salivary gland basal lamina as a selective barrier associated with vector-specific transmission of barley yellow dwarf luteoviruses. Phytopathology 83:1293-1302.

14. Gildow, F. E., and Rochow, W. F. 1980. Importance of capsid integrity for interference between two isolates of barley yellow dwarf virus in an aphid. Phytopathology 70:1013-1015.

15. Gomez-Machorro, C., Bennett, K. E., del Lourdes Munoz, M., and Black, W. C. 2004. Quantitative trait loci affecting dengue midgut infection barriers in an advanced intercross line of Aedes aegypti. Insect Mol. Biol. 13:637-648.

16. Gray, S. M., and Banerjee, N. 1999. Mechanisms of arthropod transmission of plant and animal viruses. Microbiol. Mol. Biol. Rev. 63:128148.

17. Gray, S. M., Chapin, J. W., Smith, D. M., Banerjee, N., and Thomas, J. S. 1998. Barley yellow dwarf luteoviruses and their predominant aphid vectors in winter wheat grown in South Carolina. Plant Dis. 82:1328-1333.

18. Gray, S. M., and Gildow, F. E. 2003. Luteovirus-aphid interactions. Annu. Rev. Phytopathol. 41:539-566.

19. Gray, S. M., Power, A. G., Smith, D. M., Seaman, A. J., and Altman, N. S 1991. Aphid transmission of barley yellow dwarf virus: Acquisition access periods and virus concentration requirements. Phytopathology 81:539-545.

20. Gray, S. M., Smith, D. M., Barbierri, L., and Burd, J. 2002. Virus transmission phenotype is correlated with host adaptation among genetically diverse populations of the aphid Schizaphis graminum. Phytopathology 92:970-975

21. Gubler, D. J., Nalim, S., Tan, R., Saipan, H., and Saroso, J. S. 1979. Variation in susceptibility to oral infection with dengue viruses among geographic strains of Aedes aegypti. Am. J. Trop. Med. Hyg. 28:10451052.

22. Gubler, D. J., and Rosen, L. 1976. Variation among geographic strains of Aedes albopictus in susceptibility to infection with dengue viruses. Am. J. Trop. Med. Hyg. 25:318-325.

23. Guo, J.-Q., Lapierre, H., and Moreau, J.-P. 1997. Vectoring ability of aphid clones of Rhopalosiphum padi (L.) and Sitobion avenae (Fabr.) and their capacity to retain barley yellow dwarf virus. Ann. Appl. Biol. 131:179-188.

24. Guo, J. Q., Lapierre, H., and Moreau, J. P. 1997. Clonal variations and virus regulation by aphids in transmission of a French PAV-type isolate of Barley yellow dwarf virus. Plant Dis. 81:570-575.

25. Hammond, J., Lister, R., and Foster, J. 1983. Purification, identity and some properties of an isolate of barley yellow dwarf virus from Indiana. J. Gen. Virol. 64:667-676.

26. Hardy, J. L., Apperson, G., Asman, S. M., and Reevers, W. C. 1978. Selection of a strain of Culex tarsalis highly resistant to infection following ingestion of western equine encephalomyelitis virus. Am. J. Trop. Med. Hyg. 27:313-321.

27. Katsar, C., and Gray, S. 1999. Rearing aphids to use in virus-vector studies. Pages 183-195 in: Maintenance of Animal/Human and Plant Pathogen Vectors. K. Maramorosch and F. Mahmood, eds. Science Publishers, Enfield, NH.

28. Katsar, C., Paterson, A., Teetes, G., and Peterson, G. 2002. Molecular analysis of sorghum resistance to the greenbug (Homoptera: Aphididae). J. Econ. Entomol. 95:448-457.

29. Li, C., Cox-Foster, D., Gray, S. M., and Gildow, F. 2001. Vector specificity of Barley yellow dwarf virus (BYDV) transmission: Identification of potential cellular receptors binding BYDV-MAV in the aphid, Sitobion avenae. Virology 286:125-133.

30. Loker, E. S. 2004. Invertebrate immune systems-not homogeneous, not simple, not well understood. Immunol. Rev. 198:10-24.

31. Lushal, G., Barro, P. J. D., David, O., Sherratt, T. N., and Maclean, N. 1998. Genetic variation within a parthenogenetic lineage. Insect Mol. Biol. 7:337-344.

32. McCullagh, P., and Nelder, J. A. 1989. Generalized Linear Models. Chapman and Hall, London.

33. Miller, B. R., and Mitchell, C. J. 1991. Genetic selection of a flavivirusrefractory strain of the yellow fever mosquito Aedes aegypti. Am. J. Trop. Med. Hyg. 45:399-407.

34. Miller, W. A., and Rasochova, L. 1997. Barley yellow dwarf viruses. Annu. Rev. Phytopathol. 35:167-190. 
35. Morlais, I., Mori, A., Schneider, J. R., and Severson, D. W. 2003. A targeted approach to the identification of candidate genes determining susceptibility to Plasmodium gallinaceum in Aedes aegypti. Mol. Gen. Genomics 269:753.

36. Papura, D., Jacquot, E., Dedryver, C. A., Luche, S., Riault, G., Bossis, M., and Rabilloud, T. 2002. Two-dimensional electrophoresis of proteins discriminates aphid clones of Sitobion avenae differing in BYDV-PAV transmission. Arch. Virol. 147:1881-1898.

37. Peiffer, M. L., Gildow, F. E., and Gray, S. M. 1997. Two distinct mechanisms regulate luteovirus transmission efficiency and specificity at the aphid salivary gland. J. Gen. Virol. 78:495-503.

38. Pereira, A. M. N., Lister, R. M., Barbara, D. J., and Shaner, G. E. 1989. Relative transmissibility of barley yellow dwarf virus from sources with differing virus contents. Phytopathology 79:1353-1358.

39. Porter, D. R., Burd, J. D., Shufran, K. A., Webster, J. A., and Teetes, G. 1997. Greenbug (Homoptera: Aphididae) biotypes: Selected by resistant cultivars or preadapted opportunists? J. Econ. Entomol. 90:1055-1065.

40. Power, A. G., and Gray, S. M. 1995. Aphid transmission of barley yellow dwarf viruses: Interactions between viruses, vectors, and host plants. Pages 259-291 in: Barley Yellow Dwarf: 40 Years of Progress. C. J. D'Arcy and P. A. Burnett, eds. The American Phytopathological Society, St. Paul, MN.

41. Puterka, G. J., and Peters, D. C. 1995. Genetics of greenbug (Homoptera, Aphididae) virulence to resistance in sorghum. J. Econ. Entomol. 88:421429.

42. Puterka, G. J., and Slosser, J. E. 1983. Inducing oviparae and males of biotype-C greenbugs, Schizaphis graminum (Rond) (Homoptera, Aphidadae). Southwest. Entomol. 8:268-272.

43. Rider, S. D., Wilde, G. E., and Kambhampati, S. 1998. Genetics of esterase-mediated insecticide resistance in the aphid Schizaphis graminum. Heredity 81:14-19.

44. Rochow, W. F., and Pang, E. 1961. Aphids can acquire strains of barley yellow dwarf virus they do not transmit. Virology 15:382-384.

45. Rouze-Jouan, J., Terradot, L., Pasquer, F., Tanguy, S., and Giblot DucrayBourdin, D. 2001. The passage of Potato leafroll virus through Myzus persicae gut membrane regulates transmission efficiency. J. Gen. Virol.
82:17-23.

46. Seddas, P., Biossinot, S., Strub, J.-M., Dorsselaer, A. V., Regenmortel, M. H. V. V., and Pattus, F. 2004. Rack-1, GAPDH3, and actin: Proteins of Myzus persicae potentially involved in the transcytosis of Beet western yellows virus particles in the aphid. Virology 325:399-412.

47. Storey, H. H. 1932. The inheritance by an insect vector of the ability to transmit a plant virus. Proc. R. Soc. London, Ser. B 112:46-60.

48. Storey, H. H. 1933. Investigations of the mechanism of the transmission of plant viruses by insect vectors - I. Proc. R. Soc. London, Ser. B 113:463-485.

49. Tabachnick, W. J. 1991. Genetic control of oral susceptibility to infection of Culicoides variipennis with bluetongue virus. Am. J. Trop. Med. Hyg. 45:666-671.

50. Tabachnick, W. J. 1994. Genetics of insect vector competence for arboviruses. Adv. Dis. Vector Res. 10:93-108.

51. Tardieux, I., Poupel, O., Lapchin, and Rodhain, F. 1991. Analysis of inheritance of oral susceptibility of Aedes aegypti to dengue-2 virus using isofemale lines. J. Med. Entomol. 28:518-521.

52. van den Heuvel, J. F. J. M., Bruyere, A., Hogenhout, S. A., Ziegler-Graff, V., Brault, V., Verbeek, M., van der Wilk, F., and Richards, K. 1997. The $\mathrm{N}$-terminal region of the luteovirus readthrough domain determines virus binding to Buchnera GroEL and is essential for virus persistence in the aphid. J. Virol. 71:7258-7265.

53. van den Heuvel, J. F. J. M., Verbeek, M., and van der Wilk, F. 1994 Endosymbiotic bacteria associated with circulative transmission of potato leafroll virus by Myzus persicae. J. Gen. Virol. 75:2559-2565.

54. Via, S. 1992. Inducing the sexual forms and hatching the eggs of pea aphids. Entomol. Exp. Appl. 65:119-127.

55. Via, S. E. 1991. The genetic structure of host plant adaptation in a spatial patchwork: Demographic variability among reciprocally transplanted pea aphid clones. Evolution 45:827-852.

56. Washburn, J., Kirkpatrick, B., and Volkman, L. 1996. Insect protection against viruses. Nature 383:767-767.

57. Zeigler, R. S., and Morales, F. J. 1990. Genetic determination of replication of rice hoja blanca virus within its planthopper vector, Sogatodes oryzicola. Phytopathology 80:559-566. 